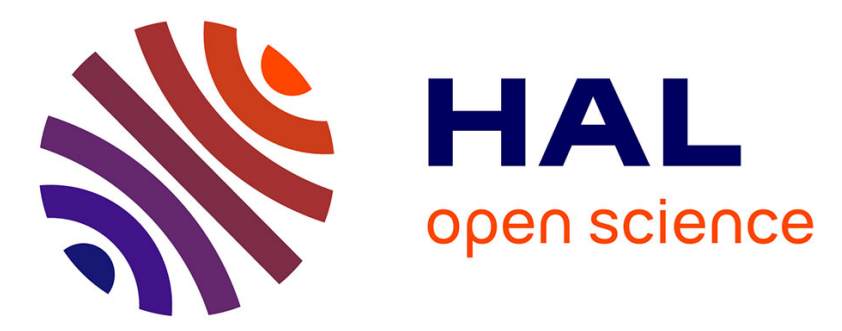

\title{
Differentials and Semidifferentials for Metric Spaces of Shapes and Geometries
}

\author{
Michel C. Delfour
}

\section{To cite this version:}

Michel C. Delfour. Differentials and Semidifferentials for Metric Spaces of Shapes and Geometries. 27th IFIP Conference on System Modeling and Optimization (CSMO), Jun 2015, Sophia Antipolis, France. pp.230-239, 10.1007/978-3-319-55795-3_21. hal-01626899

\section{HAL Id: hal-01626899 \\ https://hal.inria.fr/hal-01626899}

Submitted on 31 Oct 2017

HAL is a multi-disciplinary open access archive for the deposit and dissemination of scientific research documents, whether they are published or not. The documents may come from teaching and research institutions in France or abroad, or from public or private research centers.
L'archive ouverte pluridisciplinaire HAL, est destinée au dépôt et à la diffusion de documents scientifiques de niveau recherche, publiés ou non, émanant des établissements d'enseignement et de recherche français ou étrangers, des laboratoires publics ou privés. 


\title{
Differentials and Semidifferentials for Metric Spaces of Shapes and Geometries
}

\author{
Michel C. Delfour ${ }^{\star}$ \\ Centre de recherches mathématiques and \\ Département de mathématiques et de statistique, \\ Université de Montréal, CP 6128, succ. Centre-ville, Montréal (Qc), Canada H3C 3J7 \\ delfour@crm. umontreal.ca \\ http://dms. umontreal.ca/ delfour/
}

\begin{abstract}
The Hadamard semidifferential retains the advantages of the differential calculus such as the chain rule and semiconvex functions are Hadamard semidifferentiable. The semidifferential calculus extends to subsets of $\mathbb{R}^{n}$ without Euclidean smooth structure. This set-up is an ideal tool to study the semidifferentiability of objective functions with respect to families of sets which are non-linear non-convex complete metric spaces. Shape derivatives are differentials for spaces endowed with Courant metrics. Topological derivatives are shown to be semidifferentials on the group of Lebesgue measurable characteristic functions.
\end{abstract}

Keywords: Semidifferential, shape and topological derivatives

\section{Introduction}

In the past decades, direct constructions of complete metric spaces of shapes and geometries (cf., for instance, M. C. Delfour and J.-P. Zolésio [7]) and, additional new ones (cf., M. C. Delfour [6]) have been given without appealing to the classical notions of atlases or smooth manifolds encountered in classical Differential Geometry. Since, at best, such spaces are groups, the issue of making sense of tangent spaces and differentials naturally arises not only for "differentiable" functions but also for large classes of "non-differentiable" functions.

In that context, the geometrical definition of a differentiable function of $\mathrm{J}$. Hadamard [11] is especially interesting since it implicitly involves the construction of trajectories (or paths) and tangent vectors to trajectories living in the space under investigation. His definition was relaxed by M. Fréchet [10] in 1937 by dropping the requirement that the differential be linear with respect to the direction or tangent vector while preserving two important properties of the differential calculus: the continuity of the function and the chain rule. A vast litterature on differentials on topological spaces followed (cf., for instance, the survey papers of V. I. Averbuh and O. G. Smoljanov [3] in 1988 and the 207page paper of M. Z. Nashed [12] for a rather complete account until 1971). The

\footnotetext{
* This research has been supported by a Discovery Grant from the Natural Sciences and Engineering Research Council of Canada.
} 
definition of Fréchet can be further relaxed to the one of semidifferential which handles convex and semiconvex functions while preserving the two properties.

Since the semidifferential is not required to be linear, they have far reaching consequences for a function $f: A \rightarrow B$ between arbitrary sets $A$ and $B$. De facto, this relaxes the requirement that the tangent spaces in each points of the sets $A$ and $B$ be linear spaces. It is sufficient to work with tangent cones to $A$ and $B$ such as Bouligand's tangent cone to make sense of semidifferentials. Shortcircuiting the requirement of a smooth manifold makes it possible to directly study the tangent spaces to non-convex metric spaces of shapes and geometries.

We show that the metric group of Lebesgue measurable characteristic functions has semi-tangents and that the notion of topological derivative of J. Sokołowski and A. Zóchowski [14] is in fact a semidifferential obtained by dilatation of a point creating a hole. By extending this construction via dilatations, we also show that the tangent space contains distributions creating topological perturbations along curves and surfaces that can break the connectivity of the set. In the same spirit dilatations of $d$-rectifiable and some $H_{d}$-rectifiable compact sets $\left(H_{d}, d\right.$-dimensional Hausdorff measure) of Ambrosio et al [1] also generate semi-tangents. Orthogonal dilatations of closed subsets of the boundary of a set of positive reach can also be used via Steiner formula (see Federer [8]).

\section{Hadamard Differential and Semidifferential}

Hadamard Differential. In 1923 J. Hadamard [11] gave a geometrical definition by using an auxiliary function $t \mapsto x(t): \mathbb{R} \rightarrow \mathbb{R}^{N}$ such that

$$
x(0)=a \quad \text { and } \quad x^{\prime}(0) \stackrel{\text { def }}{=} \lim _{t \rightarrow 0} \frac{x(t)-a}{t} \text { exists in } \mathbb{R}^{\mathrm{N}},
$$

where $\mathbb{R}$ is the field of real numbers. It defines a path that induces a perturbation or a variation of the point $a$. We shall use the terminology time for the auxiliary variable $t$ and admissible trajectory for the auxiliary function $x$. Note that $x$ need not be continuous or differentiable at $t \neq 0$. The vector $x^{\prime}(0)$ is the tangent to the trajectory $x$ at the point $x(0)=a$. Scaling $t$ by an arbitrary non-zero real number generates a whole line tangent to $x$ at $a$

Definition 1. A function $f: \mathbb{R}^{N} \rightarrow \mathbb{R}^{K}$ is Hadamard differentiable at $a \in \mathbb{R}^{N}$ if

(i) for all admissible trajectories $x$ at $a$ the limit

$$
(f \circ x)^{\prime}(0) \stackrel{\text { def }}{=} \lim _{t \rightarrow 0} \frac{f(x(t))-f(a)}{t} \text { exists in } \mathbb{R}^{K}
$$

(ii) and there exists a linear function $D f(a): \mathbb{R}^{N} \rightarrow \mathbb{R}^{K}$ such that for all admissible trajectories $x$ at $a$

$$
(f \circ x)^{\prime}(0)=D f(a)\left(x^{\prime}(0)\right) .
$$

$D f(a)$ is the differential of $f$ at $a$. 
The definition of Hadamard differentiability is equivalent to the one of Fréchet differentiability in finite dimension. In Banach and Fréchet spaces, a Hadamard differentiable function at a point $a$ is continuous at $a$ and the chain rule is applicable. In 1937, Fréchet [10] insisted on the fact that the definition of Hadamard is more general than his since it extends to functions $f: X \rightarrow \mathbb{R}^{K}$ defined on topological vector spaces $X$ that are not normed vector spaces. Furthermore, in Banach spaces of functions, we can consider the set of tangent vectors (functions) $x^{\prime}(0)$ as weak limits ... and even as distributions.

In his 1937 paper, Fréchet [10] observed that, in function spaces, the Hadamard differentiability is not only a notion more general than the one he introduced in 1911 but that the linearity in part (ii) is not necessary to preserve the continuity of the function and the chain rule. He gives the following example:

$$
f\left(x_{1}, x_{2}\right) \stackrel{\text { def }}{=} x \sqrt{\frac{x_{1}^{2}}{x_{1}^{2}+x_{2}^{2}}} \quad \text { for }\left(x_{1}, x_{2}\right) \neq(0,0) \text { and } \quad f(0,0) \stackrel{\text { def }}{=} 0 .
$$

([10, p. 239]). Indeed, it is readily checked that for any trajectory $x: \mathbb{R}^{2} \rightarrow \mathbb{R}$ such that $x(0)=(0,0)$ and $x^{\prime}(0)$ exists

$$
(f \circ x)^{\prime}(0) \stackrel{\text { def }}{=} \lim _{t \rightarrow 0} \frac{f(x(t))-f(0,0)}{t}=f\left(x^{\prime}(0)\right) .
$$

Hadamard always insisted on the linearity and this new notion was criticized by P. Lévy. Yet, his example shows that such nondifferentiable functions exist.

Hadamard Semidifferential. By relaxing the linearity, we can deal with some families of non-differentiable functions. Unfortunately, some convex continuous functions and, in particular, the norm $\|x\|$ in $a=0$, are not differentiable in this relaxed sense. To get around this, we need the notion of semidifferential.

For instance, in the case of the Euclidean norm $x \mapsto f(x)=\|x\|: \mathbb{R}^{\mathrm{N}} \rightarrow \mathbb{R}$ at $x=0$, consider a semi-trajectory $x:[0,+\infty) \rightarrow \mathbb{R}^{N}$ through the origin $x(0)=0$ for which the right-hand limit $x^{\prime}\left(0^{+}\right)$exists. We get at $a=0$

$$
(f \circ x)^{\prime}\left(0^{+}\right) \stackrel{\text { def }}{=} \lim _{t \searrow 0} \frac{f(x(t))-f(0)}{t}=\lim _{t \searrow 0}\left\|\frac{x(t)-x(0)}{t}\right\|=\left\|x^{\prime}\left(0^{+}\right)\right\|,
$$

where the notation $t \searrow 0$ means that $t$ goes to 0 by strictly positive values. We have a similar result for convex and semiconvex continuous functions. When $(f \circ x)^{\prime}\left(0^{+}\right)$is not a linear function of the semi-tangent $x^{\prime}\left(0^{+}\right)$, we say that the function is semidifferentiable.

From Linear to Non-convex Spaces. The hypothesis of linearity of the differential is also a severe restriction to define a differential for a function $f: A \subset \mathbb{R}^{N} \rightarrow B \subset \mathbb{R}^{K}$ since it requires that the tangent space to $A$ at $a$ and the tangent space to $B$ at $f(a)$ be linear subspaces. This necessitates that 
the sets $A$ and $B$ be sufficiently smooth in the sense that, at each point of $A$ and of $B$, the tangent spaces be linear subspaces of $\mathbb{R}^{\mathrm{N}}$ and $\mathbb{R}^{K}$.

Since the Hadamard semidifferential does not require the linearity of the tangent space, the a priori smoothness assumption of the sets $A$ and $B$ can be de facto dropped since the semidifferential only needs to be defined on a tangent cone. Several tangent cones are available in the literature, but the following one is especially well suited for semidifferentials.

Definition 2. The Bouligand tangent cone to a set $A$ at a point $a \in \bar{A}$ is

$$
T_{a} A \stackrel{\text { def }}{=}\left\{v \in \mathbb{R}^{N}: \exists\left\{x_{n}\right\} \subset A \text { and }\left\{t_{n} \searrow 0\right\} \text { such that } \lim _{n \rightarrow \infty} \frac{x_{n}-a}{t_{n}}=v\right\} .
$$

When the boundary $\partial A$ of $A$ is smooth, $T_{a} A$ is a linear subspace of $\mathbb{R}^{N}$. However,

$$
\begin{aligned}
& x^{\prime}(0) \stackrel{\text { def }}{=} \lim _{t \rightarrow 0} \frac{x(t)-a}{t} \text { exists } \\
& \text { - tangent linear subspace } T_{a}(A)
\end{aligned}
$$

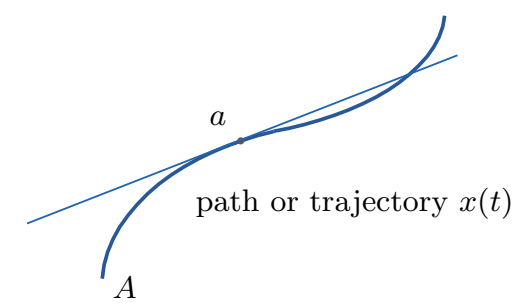

the linearity of $T_{a} A$ puts a severe restriction on the sets $A$. For instance, the requirement that $T_{a} A$ be linear rules out a curve in $\mathbb{R}^{2}$ with kinks.

- tangent (non convex) cone $T_{a}(A)$

$x^{\prime}\left(0^{+}\right) \stackrel{\text { def }}{=} \lim _{t \searrow 0} \frac{x(t)-a}{t}$ exists

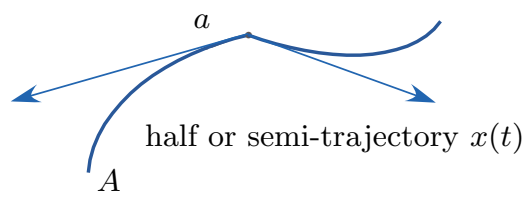

This naturally leads to the following notions of admissible trajectory.

Definition 3. Given $A \subset \mathbb{R}^{\mathrm{N}}$, an admissible semi-trajectory in $A$ at $a \in \bar{A}$ is a function $x:[0, \tau] \rightarrow A, \tau>0$, such that the semi-tangent at $a$

$$
x^{\prime}\left(0^{+}\right) \stackrel{\text { def }}{=} \lim _{t \searrow 0} \frac{x(t)-a}{t}
$$

exists. When the limit $x^{\prime}\left(0^{+}\right)$exists, it follows that $x(t) \rightarrow a$ as $t \searrow 0$.

An equivalent characterization of the Bouligand's tangent cone is obtained.

Theorem 1. $T_{a} A=\left\{x^{\prime}\left(0^{+}\right): x\right.$ is an admissible semi-trajectory in $A$ at $\left.a\right\}$.

Following Fréchet, we now relax the linearity and formalize the notion of semidifferential for functions $f: A \rightarrow B$. 
Definition 4 (Geometrical definition). Given $A \subset \mathbb{R}^{\mathrm{N}}$ and $B \subset \mathbb{R}^{K}$, the function $f: A \rightarrow B$ is Hadamard semidifferentiable at $a \in A$ if

(i) for each admissible semi-trajectory $x$ in $A$ at $a$, the limit

$$
(f \circ x)^{\prime}\left(0^{+}\right) \stackrel{\text { def }}{=} \lim _{t \searrow 0} \frac{f(x(t))-f(a)}{t} \text { exists }
$$

(ii) and there exists a (positively homogeneous) function $v \mapsto d_{A} f(a ; v): T_{a} A \rightarrow$ $T_{f(a)} B$ such that for all admissible semi-trajectories $x$ in $A$ at $a$

$$
(f \circ x)^{\prime}\left(0^{+}\right)=d_{A} f\left(a ; x^{\prime}\left(0^{+}\right)\right) .
$$

The function $v \mapsto d_{A} f(a)(v)=d_{A} f(a ; v)$ is referred to as the (tangential) semidifferential of $f$ at $a \in A$. It can be shown that $d_{A} f(a)$ is continuous on $T_{a} A$.

This definition has an equivalent analytical counterpart.

Theorem 2 (Analytical definition). Given $A \subset \mathbb{R}^{\mathrm{N}}$ and $B \subset \mathbb{R}^{K}$, the function $f: A \rightarrow B$ is Hadamard semidifferentiable at $a \in A$ if and only if there exists a (positively homogeneous) function $v \mapsto d_{A} f(a ; v): T_{a} A \rightarrow T_{f(a)} B$ such that for all $v \in T_{a} A$ and all sequences $\left\{x_{n}\right\} \subset A$ and $\left\{t_{n} \searrow 0\right\}$ such that $\left(x_{n}-a\right) / t_{n} \rightarrow v$

$$
\lim _{n \rightarrow \infty} \frac{f\left(x_{n}\right)-f(a)}{t_{n}}=d_{A} f(a ; v) .
$$

With the above definitions, the two important properties are preserved: continuity of $f$ at $a$ and the chain rule. The previous definitions extend to subsets $A$ of topological vector spaces $X$, but we have to be careful and retain the abstract notions that are really meaningful. For shapes and geometries, the subset $A$ will be a complete metric space with or without a group structure in a surrounding Banach or Fréchet space. We consider Courant metrics and the metric space of characteristic functions. Oriented distance functions can also be considered.

\section{Metric Group of Characteristic Functions}

Consider the metric Abelian group of characteristic functions on $\mathbb{R}^{\mathrm{N}}$

$$
\mathrm{X}\left(\mathbb{R}^{\mathrm{N}}\right)=\left\{\chi_{\Omega}: \Omega \subset \mathbb{R}^{\mathrm{N}} \text { Lebesgue measurable }\right\} \subset L^{\infty}\left(\mathbb{R}^{\mathrm{N}}\right) .
$$

It is a closed subset without interior of the Banach space $L^{\infty}\left(\mathbb{R}^{\mathrm{N}}\right)$ and of the Fréchet spaces $L_{\text {loc }}^{p}\left(\mathbb{R}^{\mathrm{N}}\right), 1 \leq p<\infty$. The analog would be the sphere in $\mathbb{R}^{3}$.

\subsection{Velocity Method}

For the velocity method, consider the following continuous trajectory in $X\left(\mathbb{R}^{\mathrm{N}}\right)$

$$
t \mapsto \chi_{T_{t}(V)(\Omega)}:[0,1] \rightarrow X\left(\mathbb{R}^{\mathrm{N}}\right), \quad \frac{d T_{t}(V)}{d t}=V(t) \circ T_{t}(V), T_{0}(V)=I .
$$


The semitangent at $\chi_{\Omega}$ is obtained by considering the limit of the differential quotient $\left(\chi_{T_{t}(V)(\Omega)}-\chi_{\Omega}\right) / t \in L^{\infty}\left(\mathbb{R}^{\mathrm{N}}\right)$ which does not exist in $L^{\infty}\left(\mathbb{R}^{\mathrm{N}}\right)$, but also not in $L_{\text {loc }}^{p}\left(\mathbb{R}^{N}\right), 1 \leq p<\infty$.

To get a derivative consider the distribution associated with $\chi_{T_{t}(V)(\Omega)}$

$$
\phi \mapsto \int_{\mathbb{R}^{\mathrm{N}}} \chi_{T_{t}(V)(\Omega)} \phi d x=\int_{T_{t}(V)(\Omega)} \phi d x=\int_{\Omega} \phi \circ T_{t} \operatorname{det} D T_{t} d x: \mathcal{D}\left(\mathbb{R}^{\mathrm{N}}\right) \rightarrow \mathbb{R}
$$

If $V \in C^{0,1}\left(\overline{\mathbb{R}^{N}}, \mathbb{R}^{N}\right)$, then

$$
\left.\frac{d}{d t}\right|_{t=0^{+}} \int_{\Omega} \phi \circ T_{t} \operatorname{det} D T_{t} d x=\int_{\Omega} \operatorname{div}(V(0) \phi) d x=\int_{\mathbb{R}^{\mathbb{N}}} \chi_{\Omega} \operatorname{div}(V(0) \phi) d x
$$

(see, for instance, [7, Thm. 4.1, Chapter 9, p. 483]). The bilinear function

$$
(\phi, V) \mapsto \int_{\mathbb{R}^{\mathrm{N}}} \chi_{\Omega} \operatorname{div}(V(0) \phi) d x: H_{0}^{1}\left(\mathbb{R}^{\mathrm{N}}\right) \times C^{0,1}\left(\overline{\mathbb{R}^{\mathrm{N}}}, \mathbb{R}^{\mathrm{N}}\right) \rightarrow \mathbb{R}
$$

is continuous. This generates the continuous linear mapping $V \mapsto \nabla \chi_{\Omega} \cdot V$ : $C^{0,1}\left(\overline{\mathbb{R}^{\mathrm{N}}}, \mathbb{R}^{\mathrm{N}}\right) \rightarrow H^{-1}\left(\mathbb{R}^{\mathrm{N}}\right)$

$$
\left(\nabla \chi_{\Omega} \cdot V\right) \phi \stackrel{\text { def }}{=} \int_{\mathbb{R}^{\mathrm{N}}} \chi_{\Omega} \operatorname{div}(V(0) \phi) d x,
$$

where $\nabla \chi_{\Omega}$ is the distributional gradient of $\chi_{\Omega}$. The support of $\nabla \chi_{\Omega} \cdot V$ is in $\Gamma$, the boundary of $\Omega$. So, the tangent space to $X\left(\mathbb{R}^{\mathrm{N}}\right)$ (considered as a subset of the space of distributions) at $\chi_{\Omega}$ contains the linear subspace

$$
\left\{\nabla \chi_{\Omega} \cdot V: V \in C^{0,1}\left(\overline{\mathbb{R}^{N}}, \mathbb{R}^{N}\right)\right\} \subset H^{-1}\left(\mathbb{R}^{N}\right) \subset \mathcal{D}\left(\mathbb{R}^{N}\right)^{\prime}
$$

of functions in $H^{-1}\left(\mathbb{R}^{\mathrm{N}}\right)$. When $\Omega$ is an open set with Lipschitz boundary

$$
\left.\frac{d}{d t}\right|_{t=0^{+}} \int_{\mathbb{R}^{\mathrm{N}}} \chi_{T_{t}(V)(\Omega)} \phi d x=\int_{\Gamma} V(0) \cdot n_{\Gamma} \phi d H_{N-1}
$$

is a bounded measure, where $H_{d}$ is the $d$-dimensional Hausdorff measure.

\subsection{Topological Derivative via Dilatations}

The rigorous introduction of the topological derivative in 1999 by Sokołowski and Zochowski [14]) (see also the book by Novotny-Sokołowski [13]) opened a broader spectrum of notions of "differential" with respect to a set. The set $\Omega$ is topologically perturbed by introducing a small hole around a point $a \in \Omega$, that is, a dilatation of $a$. This idea can be readily extended to some families of closed subsets $E$ of $\Omega$ of dimension $d, 1 \leq d \leq N-1$, for which $H_{d}(E)$ is finite.

Given $\Omega \subset \mathbb{R}^{N}$ open, we consider several examples where $\mathrm{m}_{N}$ denotes the Lebesgue measure in $\mathbb{R}^{N}$. The distance function $d_{E}(x)$ of $x$ to a subset $E \subset \mathbb{R}^{N}$ and the $r$-dilatation of $E$ are defined as

$$
d_{E}(x) \stackrel{\text { def }}{=} \inf _{y \in E}|x-y|, \quad E_{r} \stackrel{\text { def }}{=}\left\{x \in \mathbb{R}^{N}: d_{E}(x) \leq r\right\} .
$$


Example 1. $E=\{a\}, a \in \mathbb{R}^{3}, \operatorname{dim} E=0$. The $r$-dilatation of $E$ is $\bar{B}_{r}(a)$,

$$
\begin{gathered}
t \stackrel{\text { def }}{=} \mathrm{m}_{3}\left(\bar{B}_{r}(a)\right)=\alpha_{3} r^{3}, \quad \alpha_{3}=4 \pi / 3=\text { volume of unit ball in } \mathbb{R}^{3} \\
\phi \mapsto \phi(a): \mathcal{D}\left(\mathbb{R}^{3}\right) \rightarrow \mathbb{R} \text { is a distribution. }
\end{gathered}
$$

Assuming that $\bar{B}_{r}(a) \subset \Omega$ for some $r>0$, the perturbed sets will be

$$
t \mapsto \Omega_{t} \stackrel{\text { def }}{=} \Omega \backslash E_{r}=\Omega \backslash \bar{B}_{\sqrt[3]{t / \alpha_{3}}}(a) .
$$

Given $\phi \in \mathcal{D}\left(\mathbb{R}^{3}\right)$, the weak limit of the differential quotient $\left(\chi_{\Omega_{t}}-\chi_{\Omega}\right) / t$ is

$$
\begin{aligned}
\frac{1}{t}\left[\int_{\Omega_{t}} \phi d x-\int_{\Omega} \phi d x\right] & =-\frac{1}{\mathrm{~m}_{3}\left(\bar{B}_{\left.\sqrt[3]{t / \alpha_{3}}\right)}(a)\right)} \int_{\bar{B}_{\sqrt[3]{t / \alpha_{3}}}(a)} \chi_{\Omega} \phi d x \\
& =-\frac{1}{\mathrm{~m}_{3}\left(\bar{B}_{r}(a)\right)} \int_{\bar{B}_{r}(a)} \chi_{\Omega} \phi d x \rightarrow-\phi(a) .
\end{aligned}
$$

This distribution is a half tangent since for all $\rho>0$

$$
\frac{1}{t}\left[\int_{\Omega_{\rho t}} \phi d x-\int_{\Omega} \phi d x\right] \rightarrow-\rho \phi(a) .
$$

Example 2. Let $A \subset \mathbb{R}^{3}$ be an open set of class $C^{1,1}, \partial A$ compact, $\operatorname{dim} \partial A=2$, and $b_{A}(x) \stackrel{\text { def }}{=} d_{A}(x)-d_{\mathbb{R}^{3} \backslash A}(x)$ be the oriented distance function, then

$\exists \varepsilon>0$ such that $b_{A} \in C^{1,1}\left(U_{\varepsilon}(\partial A)\right), \quad U_{\varepsilon}(\partial A) \stackrel{\text { def }}{=}\left\{x \in \mathbb{R}^{3}:\left|b_{A}(x)\right|<\varepsilon\right\}$

projection onto $\partial A: p_{\partial A}(x)=x-b_{A}(x) \nabla b_{A}(x), \quad H_{2}(\partial A)<\infty$.

Consider the shell or sandwich of thickness $t=2 r$ around $E=\partial A$ and, for $0<$ $r<\varepsilon$, the $r$-dilatation $E_{r} \stackrel{\text { def }}{=}\left\{x \in \mathbb{R}^{3}:\left|b_{A}(x)\right| \leq r\right\}=\left\{x \in \mathbb{R}^{3}: d_{\partial A}(x) \mid \leq r\right\}$,

$$
\begin{gathered}
t=2 r=\alpha_{1} r, \quad \alpha_{1}=2=\text { volume of the unit ball in } \mathbb{R}^{1} \\
\phi \mapsto \int_{E} \phi d H_{2}: \mathcal{D}\left(\mathbb{R}^{3}\right) \rightarrow \mathbb{R} \text { is a distribution. }
\end{gathered}
$$

Assuming that $U_{\varepsilon}(\partial A) \subset \Omega$, the perturbed sets for $0<t<\varepsilon$ are

$$
t \mapsto \Omega_{t} \stackrel{\text { def }}{=} \Omega \backslash E_{r}=\Omega \backslash E_{t / 2} .
$$

Given $\phi \in \mathcal{D}\left(\mathbb{R}^{3}\right)$, the weak limit of the differential quotient $\left(\chi_{\Omega_{t}}-\chi_{\Omega}\right) / t$ is

$$
\begin{aligned}
\frac{1}{t}\left[\int_{\Omega_{t}} \phi d x-\int_{\Omega} \phi d x\right] & =-\frac{1}{t} \int_{E_{t / 2}} \chi_{\Omega} \phi d x \\
& =-\frac{1}{\alpha_{1} r} \int_{E_{r}} \chi_{\Omega} \phi d x \rightarrow-\int_{E} \phi d H_{2} .
\end{aligned}
$$


This distribution is a half tangent since for all $\rho>0$

$$
\frac{1}{t}\left[\int_{\Omega_{\rho t}} \phi d x-\int_{\Omega} \phi d x\right] \rightarrow-\rho \int_{E} \phi d H_{2} .
$$

When $E=\partial A$, we create a new connected component (cf. Figure 1). This

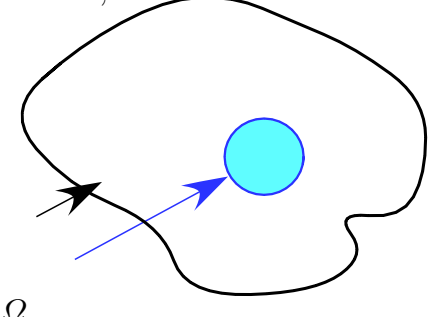

$\bar{A}$

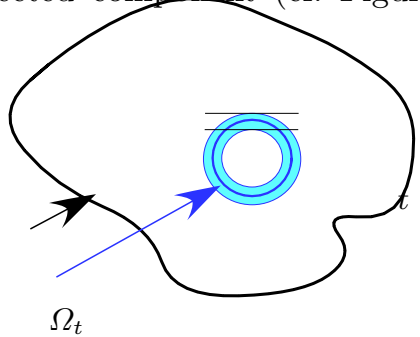

$(\partial A)_{t / 2}$

Fig. 1. For $E=\partial A, \Omega_{t}$ has two connected components

construction extends to a set of class $C^{1,1}$ with compact boundary in $\mathbb{R}^{N}$.

Example 3. Let $A=\partial A \subset \mathbb{R}^{N}, N \geq 3$, be a compact $C^{2}$-submanifold for which there exists $\varepsilon>0$ such that $d_{A}^{2} \in C^{2}\left(U_{\varepsilon}(A)\right)$, then

$$
\text { projection onto } \partial A: p_{A}(x)=x-\frac{1}{2} \nabla d_{A}^{2}(x), \quad D p_{A}(x)=I-\frac{1}{2} D^{2} d_{A}^{2}(x),
$$

$\operatorname{Im} D p_{A}(x)=$ tangent space at $x \in A, \quad \operatorname{dim} A(x)=\operatorname{dim}\left(\operatorname{Im} D p_{A}(x)\right)$.

Let $\operatorname{dim} A=d$ and $H_{d}(A)<\infty$ for some $d, 0<d<N-1$. Given $E=A$ and $0<r<\varepsilon$, consider the $r$-dilatation $E_{r}$ of $E$,

$$
\begin{gathered}
t=\alpha_{N-d} r^{N-d}, \quad \alpha_{N-d}=\text { volume of the unit ball in } \mathbb{R}^{N-d} \\
\phi \mapsto \int_{E} \phi d H_{d}: \mathcal{D}\left(\mathbb{R}^{\mathcal{N}}\right) \rightarrow \mathbb{R} \text { is a distribution. }
\end{gathered}
$$

Assuming that $U_{\varepsilon}(A) \subset \Omega$, the perturbed set for $0<r<\varepsilon$ will be

$$
t \mapsto \Omega_{t} \stackrel{\text { def }}{=} \Omega \backslash E_{r}=\Omega \backslash E_{N-d} \sqrt{t / \alpha_{N-d}} .
$$

Given $\phi \in \mathcal{D}\left(\mathbb{R}^{N}\right)$, the weak limit of the differential quotient $\left(\chi_{\Omega_{t}}-\chi_{\Omega}\right) / t$ is

$$
\begin{aligned}
\frac{1}{t}\left[\int_{\Omega_{t}} \phi d \mathrm{~m}_{N}-\int_{\Omega} \phi d \mathrm{~m}_{N}\right] & =-\frac{1}{t} \int_{E_{N-d} \sqrt[d]{t / \alpha}-d} \chi_{\Omega} \phi d \mathrm{~m}_{N} \\
& =-\frac{1}{\alpha_{N-d} r^{N-d}} \int_{E_{r}} \chi_{\Omega} \phi d \mathrm{~m}_{N} \rightarrow-\int_{E} \phi d H_{d}
\end{aligned}
$$


This distribution is a half tangent since for all $\rho>0$

$$
\frac{1}{t}\left[\int_{\Omega_{\rho t}} \phi d \mathrm{~m}_{N}-\int_{\Omega} \phi d \mathrm{~m}_{N}\right] \rightarrow-\rho \int_{E} \phi d H_{d} .
$$

\section{Generalization and Concluding Remarks}

In section 3.2 we considered the Minkowski content $M^{d}(E)$ of closed subsets $E$ of $\mathbb{R}^{N}$ (of positive reach) such that

$$
M^{d}(E) \stackrel{\text { def }}{=} \lim _{r \searrow 0} \frac{m_{N}\left(E_{r}\right)}{\alpha_{N-d} r^{N-d}}=H_{d}(E), \quad 0 \leq d \leq N,
$$

and the associated distribution (measure)

$$
\phi \mapsto \int_{E} \phi d H_{d}=\lim _{r \searrow 0} \frac{1}{\alpha_{N-d} r^{N-d}} \int_{E_{r}} \phi d m_{N}: \mathcal{D}\left(\mathbb{R}^{\mathcal{N}}\right) \rightarrow \mathbb{R} .
$$

Choosing the volume $t=\alpha_{N-d} r^{N-d}$ of the ball of radius $r$ in $\mathbb{R}^{N-d}$ as the auxiliary variable, that is, $r=\left(t / \alpha_{N-d}\right)^{1 /(N-d)}$,

$$
\phi \mapsto \int_{E} \phi d H_{d}=\lim _{t \searrow 0} \frac{1}{t} \int_{E_{\left(t / \alpha_{N-d}\right)^{1 /(N-d)}}} \phi d m_{N}: \mathcal{D}\left(\mathbb{R}^{\mathcal{N}}\right) \rightarrow \mathbb{R} .
$$

Given a Lebesgue measurable $\Omega \subset \mathbb{R}^{N}$, we considered the perturbation

$$
\Omega_{t}=\Omega \backslash E_{r}
$$

and obtained a continuous trajectory $t \mapsto \chi_{\Omega_{t}}$ in $\mathrm{X}\left(\mathbb{R}^{N}\right)$ such that

$$
\chi_{\Omega_{t}} \rightarrow \chi_{\Omega \backslash E} \text { in } L_{\mathrm{loc}}^{p}\left(\mathbb{R}^{N}\right), 1 \leq p<\infty .
$$

If $m_{N}(E)=0$, then $\chi_{\Omega_{t}} \rightarrow \chi_{\Omega}$ in $L_{\mathrm{loc}}^{p}\left(\mathbb{R}^{N}\right), 1 \leq p<\infty$.

Such a construction extends to dilatations of d-rectifiable compact sets (see Federer [9]) and to $H_{d}$-rectifiable sets $E$ verifying a certain density condition (see Ambrosio et al [2, Dfn. 2.57, p. 80] and [1, pp. 730-731]).

Another family of closed sets is provided by the extension of the Steiner formula by Federer [8, Thm. 5.6, p. 455] to closed sets $A$ of positive reach. Given $E \subset \partial A$ closed and $0 \leq r<\operatorname{reach}(A)$, define the orthogonal $r$-dilatation of $E$ : $E_{r}^{A} \stackrel{\text { def }}{=}\left\{x \in \mathbb{R}^{N}: d_{A}(x) \leq r\right.$ and $\left.p_{A}(x) \in E\right\}$, where $p_{A}$ is the projection onto $A$. Then $\lim _{r \searrow 0} \mathrm{~m}_{N}\left(E_{r}^{A}\right) /\left(\alpha_{N-d} r^{N-d}\right)$ is a Radon measure for some $d, 0 \leq d \leq N$.

The emerging point of view is to consider the elements of the group $\mathrm{X}\left(\mathbb{R}^{\mathrm{N}}\right)$ of characteristic functions $\chi_{\Omega}$ of Lebesgue measurable subsets $\Omega \subset \mathbb{R}^{N}$ as a subset of measures in the space of distributions $\mathcal{D}\left(\mathbb{R}^{N}\right)^{\prime}$ :

$$
\phi \mapsto \int_{\mathbb{R}^{N}} \chi_{\Omega} \phi d x=\int_{\Omega} \phi d x: \mathcal{D}\left(\mathbb{R}^{N}\right) \rightarrow \mathbb{R}, \quad X\left(\mathbb{R}^{N}\right) \subset \mathcal{D}\left(\mathbb{R}^{N}\right)^{\prime} .
$$


It is conjectured that the tangent cone $T_{\chi_{\Omega}} \mathrm{X}\left(\mathbb{R}^{\mathrm{N}}\right)$ is contained in $\mathcal{D}\left(\mathbb{R}^{N}\right)^{\prime}$. In section 3.1 the velocities generate tangents that are distributions in $H^{1}\left(\mathbb{R}^{N}\right)^{\prime}$; in section 3.2 the compact subsets $E$ generate semi-tangents that are bounded measures. As a result, $T_{\chi_{\Omega}} \mathrm{X}\left(\mathbb{R}^{\mathrm{N}}\right)$ is not a linear space and it does not only contain measures, but we don't know how big it is. We could also attempt to characterize the tangent space to a family of measures.

\section{References}

1. L. Ambrosio, A. Colesanti, and E. Villa, Outer Minkowski content for some classes of closed sets, Math. Ann. 342 (2008), no. 4, 727-748.

2. L. Ambrosio, N. Fusco and D. Pallara, Functions of Bounded Variation and Free Discontinuity Problems, The Clarendon Press, Oxford University Press, New York, 2000.

3. V. I. Averbuh and O. G. Smoljanov, The various definitions of the derivative in linear topological spaces, (Russian) Uspehi Mat. Nauk 23 (1968) no. 4 (142) 67-113 (English Translation, Russian Math. Surveys).

4. M. C. Delfour, Groups of Transformations for Geometrical Identification Problems: Metrics, Geodesics, pp. 3403-3406, Mini-Workshop: Geometries, Shapes and Topologies in PDE-based Applications, M. Hintermüller, G. Leugering, and J. Sokolowski, organizers, Mathematisches Forschungsinstitut Oberwolfach, 2012 (Report No. 57/2012, DOI: 10.4171/OWR/2012/57).

5. M. C. Delfour, Introduction to optimization and semidifferential calculus, MOSSIAM Series on Optimization, Society for Industrial and Applied Mathematics, Philadelphia, USA, 2012.

6. M. C. Delfour, Metrics spaces of shapes and geometries from set parametrized functions, in "New Trends in Shape Optimization", A. Pratelli and G. Leugering, eds., pp. 57-101, International Series of Numerical Mathematics vol. 166, Birkhäuser Basel 2015.

7. M. C. Delfour and J.-P. Zolésio, Shapes and Geometries, metrics, analysis, differential calculus, and optimization, second edition, SIAM series on Advances in Design and Control, SIAM, Philadelphia, USA, 2011.

8. H. Federer, Curvature measures, Trans. Amer. Math. Soc. 93 (1959), 418-419.

9. H. Federer, Geometric measure theory, Die Grundlehren der mathematischen Wissenschaften, 153. Springer, New York, 1969.

10. M. Fréchet, Sur la notion de différentielle, Journal de Mathématiques Pures et Appliquées 16 (1937), 233-250.

11. J. Hadamard, La notion de différentielle dans l'enseignement, Scripta Univ. Ab. Bib., Hierosolymitanarum, Jerusalem, 1923. Reprinted in the Mathematical Gazette 19, no. 236 (1935), 341-342.

12. M. Z. Nashed, Differentiability and related properties of nonlinear operators: Some aspects of the role of differentials in nonlinear functional analysis, in "Nonlinear Functional Anal. and Appl." (ed. L. B. Rail), pp. 103-309, Academic Press, New York 1971.

13. A. A. Novotny and J. Sokołowski, Topological Derivatives in Shape Optimization, Interaction of Mech. and Math., Springer, Heidelberg, New York 2013.

14. J. Sokołowski and A. Zóchowski, On the topological derivative in shape optimization, SIAM J. Control Optim. (4) 37 (1999), 1251-1272. 